Meta

Journal des traducteurs

Translators' Journal

\title{
Informatique, traduction et enseignement des langues
}

\section{Anne-Marie Laurian}

Volume 30, numéro 3, septembre 1985

URI : https://id.erudit.org/iderudit/003420ar

DOI : https://doi.org/10.7202/003420ar

Aller au sommaire du numéro

Éditeur(s)

Les Presses de l'Université de Montréal

ISSN

0026-0452 (imprimé)

1492-1421 (numérique)

Découvrir la revue

Citer cet article

Laurian, A.-M. (1985). Informatique, traduction et enseignement des langues. Meta, 30(3), 274-279. https://doi.org/10.7202/003420ar d'utilisation que vous pouvez consulter en ligne.

https://apropos.erudit.org/fr/usagers/politique-dutilisation/ 


\section{INFORMATIQUE, TRADUCTION ET ENSEIGNEMENT DES LANGUES}

Dans le titre qui a été donné à cette journée : "La traduction dans l'enseignement des langues ", la préposition dans m'a intriguée. Fallait-il comprendre : la traduction pour l'enseignement des langues, l'enseignement de la traduction, ou l'enseignement par la traduction. Devant l'éventail des possibilités de significations de cette préposition dans (qui, pourtant, dans d'autres contextes, ne semble pas ambiguë), j'ai préféré, pour le titre de mon exposé, utiliser deux éléments de liaison qui, eux, sont bien connus pour être ambigus - sinon plurivoques ou multidimensionnels - : le et et la virgule.

Ce petit "récit autobiographique" était destiné à pointer tout de suite l'un des problèmes essentiels de la traduction : quand on se trouve devant des prépositions de grande extension comme $\grave{a}$, de, dans, par, etc., ou devant une conjonction telle que $e t$, on ne sait jamais ce qu'elle signifie, ce que l'auteur voulait exprimer ou ce qu'il a pensé en l'écrivant. Ceci est particulièrement important quand il s'agit de traitement automatique. Ainsi, par exemple, si l'on peut admettre que le correspondant anglais de et est and, et si la machine ne possède que cette règle (trop) simple, tout et français sortira en anglais comme un and. Ce qui est correct sur le plan de la surface de l'expression, mais certes insuffisant dans bien des cas car le contenu même du texte n'est pas pris en compte.

"Informatique, traduction et enseignement des langues ", pourquoi avoir posé ces trois éléments dans cet ordre ? Mon propos, ici, est de montrer comment l'informatique peut se mettre au service de la traduction, et comment la traduction, utilisant les moyens informatiques, peut servir à l'enseignement, et vice versa : comment l'enseignement pourrait servir à la traduction aidée par ordinateur ou automatique.

A cette fin, $j$ 'aborderai, après une brève description de ce qu'est la traduction automatique, les directions dans lesquelles la traduction automatique nous oblige à repenser l'enseignement des langues :

- penser en termes de quantité et non plus seulement en termes de qualité,

- différencier nettement les domaines sur lesquels on travaille,

- connaitre et utiliser les aides à la traduction tels les dictionnaires automatiques,

- préparer l'étape qui suit celle des aides : la traduction automatique avec postédition, donc préparer les étudiants à cette nouvelle activité.

Immédiatement, et pour éviter toute interprétation erronée de ce que je vais dire, je précise que je ne rejette absolument pas l'enseignement traditionnel fondé sur la culture, la civilisation, la littérature ou l'expression orale. Ces champs existent et doivent continuer à exister. Mais on voit naître en ce moment d'autres réalités qui nous ouvrent de nouveaux champs de recherche ou d'application qu'il me semble important de présenter aux étudiants.

Les champs techniques, spécialisés, de façon générale comme par exemple la documentation, ou de façon restreinte comme par exemple l'industrie, l'économie, la santé, etc., commencent à être utilisés, exploités dans l'enseignement. Ils devront prendre une place de plus en plus grande dans l'enseignement des langues. J'insiste : il faut leur donner une place de plus en plus grande dans l'enseignement supérieur comme dans l'enseignement secondaire, et ceci le plus rapidement possible - afin que ce relais ne soit pas entièrement pris par les grandes écoles ou les écoles spécialisées - Il s'agit pour les enseignants et pour les enseignements du supérieur et du secondaire d'une sorte de course à l'adaptation. En quoi consiste cette adaptation ? C'est ce que je tenterai de préciser. 
I. QU'EST-CE QUE LA TRADUCTION AUTOMATIQUE ? QUE PEUT-ELLE ? QUE NE PEUTELLE PAS ?

1. Il n'y a pas une traduction automatique mais des systèmes de traduction automatique.

Mentionnons, dans les différents pays : le GETA de Grenoble qui a un système traduisant du russe vers le français, le système SYSTRAN créé aux États-Unis pour la traduction russe-anglais et développé pour les couples anglais-français et anglais-italien dans le cadre de la Communauté européenne, les systèmes WEIDNER et ALPS créés à Provo, où d'ailleurs un troisième système est à l'étude, le système LOGOS fonctionnant aux États-Unis et en Allemagne, etc. Tous ces systèmes traduisent d'une langue nationale à une autre langue nationale sans limitation de domaine de spécialité (les dictionnaires sont en constant enrichissement). Ce sont différentes descriptions linguistiques, différentes théories qui ont mené à des productions diverses. Les résultats sont plus ou moins corrects, des fautes subsistent toujours et une post-édition est en général nécessaire.

Alors que ces systèmes fonctionnent sur les discours tels qu'ils sont, au naturel, le système TITUS, qui traduit des résumés documentaires entre le français, l'anglais, l'allemand et l'espagnol, fonctionne en "syntaxe restreinte". Cela signifie que toutes les structures linguistiques ne sont pas admises, et que les procédés morphologiques admis par l'ordinateur sont en nombre limité. Ce qui n'empêche pas l'utilisation quotidienne de ce système au Centre de documentation scientifique et technique du CNRS ainsi qu'à l'Institut textile de France - où il a d'ailleurs été créé.

Ceci nous permet de montrer comment l'usage répond à un besoin, et ce besoin étant différent selon les institutions, les réponses apportées sont différentes. Il n'y a pas de valeur plus ou moins grande d'un système par rapport à l'autre de façon intrinsèque. Il n'y a qu'une meilleure adaptation à une situation, une meilleure réponse donnée à un problème particulier.

Mentionnons encore l'Université de Sarrebrück qui a produit le système SUSY, et qui en est maintenant à la deuxième génération, un groupe aux Pays-Bas qui a imaginé un système utilisant l'espéranto comme langue-pivot, le Japon où une vingtaine de systèmes sont à l'étude.

2. Il convient de faire la différence entre $T A O$ et $T A$, autrement dit la traduction assistée par ordinateur et la traduction automatique, ce qu'en anglais on pourrait dénommer (cf. "La recherche ", décembre 83, article de M. Nagas) MAHT opposé à HAMT, c'est-àdire : Machine Aided Human Translation et Human Aided Machine Translation. Ce ne sont pas là que des sigles auxquels il nous faut nous adapter. Une simple interversion de lettres, ou l'ajout d'un $\boldsymbol{A}$ signifie un conflit théorique profond, un conflit entre groupes de recherche, équipes d'informaticiens et de linguistes, un conflit politique et économique.

En TAO, le traducteur est assis devant une console, il tape au clavier une traduction, et il demande occasionnellement à la machine une aide d'ordre terminologique ou lexicale ou des renseignements de type documentation terminologique. Il peut enrichir la mémoire d'unités lexicales ou phraséologiques dont il a lui-même observé la fréquence afin que la machine lui fournisse en bloc certains syntagmes ou certaines phrases. C'est ce qui est pratiqué par exemple chez IBM à Paris, firme où les documents techniques sont traduits ainsi.

En TA le texte à traduire est saisi par une dactylo, et la machine traite, selon des instructions-programmes prévus qui forment le système de traduction, le texte qui sort directement sur imprimante. Il y a en général des erreurs de langue à la sortie (erreurs 
de construction par exemple, lorsque les phrases sont longues), et une postédition est nécessaire. L'intervention du postéditeur est différente de celle du traducteur ou même du réviseur. Il ne s'agit pas de réécriture, sauf dans des circonstances particulières, mais de correction économique et efficace.

Ce nom de "postéditeur " qui peut sembler barbare à certains, a été créé pour différencier le travail sur sortie d'ordinateur du travail sur production humaine. La postédition n'est pas une activité une et uniforme, elle a de multiples aspects et diverses modalités (selon la fonction du texte à postéditer par exemple), mais notre propos n'est pas de développer ici cet aspect de la traduction automatique.

Entre TA et TAO, il est difficile de choisir en se plaçant sous un angle théorique ou linguistique. Les choix sont plutôt circonstanciels : chacune des méthodologies est plus ou moins bien adaptée à telle ou telle situation de production de traductions. Cependant il faut bien noter qu'il existe une lutte entre TA et TAO et que les partisansdéfenseurs de l'une ou de l'autre sont également les farouches détracteurs de l'autre. Tout chevalier-servant de Dame Traduction n'est-il pas un pourfendeur acharné de vassaux de sa rivale? Les deux technologies sont encore en évolution et seul un avenir impartial tranchera entre leurs mérites respectifs.

3. Une fois de plus, il faut préciser qu'on ne peut attendre des systèmes informatisés aucune traduction littéraire ou poétique. Les traducteurs littéraires peuvent être tranquilles : on est loin de disposer d'aucun système qui soit capable de tenir compte des sonorités, des allusions, des rythmes, des sous-entendus. La traduction automatique est utilisée et ne peut être envisagée que pour des documents techniques, scientifiques, administratifs, en un mot : spécialisés.

Ainsi, par exemple, dans le cadre de la Commission des communautés européennes, SYSTRAN est utilisé pour des notes techniques concernant la houille, la recherche de pétrole, la santé, les statistiques économiques, etc. On a tenté l'expérience de traduire un article de vulgarisation scientifique tiré de Pour la sciencel ; le vocabulaire de l'optique et de la chimie propre au domaine traité dans cet article étant absent des dictionnaires utilisés par SYSTRAN, il y a eu des lacunes ; des erreurs syntaxiques s'en sont suivies. Le système est écrit pour s'adapter à tout domaine, toute syntaxe, mais il demande à être développé, enrichi constamment, en fonction des domaines effectivement traités.

\section{CONSÉQUENCE SUR L'ENSEIGNEMENT DES LANGUES}

1. Tout ceci nous amène à repenser l'enseignement des langues. Pourquoi ?

Le principe fondamental que nous énoncerons ici est qu'il faut penser l'enseignement des langues en fonction de l'évolution des marchés professionnels et en fonction de l'évolution des techniques.

Au moment où l'on assiste à une réforme du baccalauréat, la traduction revient à l'honneur. Les épreuves de langues vivantes se modifient : on y introduit des exercices de contrôle de compétences grammaticales, des exercices de thème faisant fonction de tests grammaticaux. Ceci signifie non seulement une réintroduction d'une pensée plus abstraite (par rapport à la pratique exclusivement orale) mais également la prise en compte d'un certain rôle et d'une certaine utilisation des langues vivantes.

On réfléchit sur la finalité de l'enseignement des langues étrangères. La distinction entre compréhension et expression est formellement faite dans les nouveaux programmes du baccalauréat. Ceci peut nous amener à nous demander ce qu'il y a entre compréhension et expression ? Que se passe-t-il entre les deux ? C'est là précisément que se place le traducteur - ou la traduction. 
2. Les langues vivantes: pour quelle communication? avec qui? dans quelles situations? avec quelles intentions?

Depuis longtemps on enseigne pour que les élèves puissent communiquer d'abord avec leurs professeurs. Ensuite, à l'université, on enseigne pour que les étudiants communiquent avec les enseignants, avec les jurys de CAPES et d'agrégation. Et quand ils sont sortis de ces concours, les étudiants n'ont plus besoin de communiquer... Ils sont enseignants à leur tour, face à leurs classes, c'est maintenant aux élèves à se débrouiller pour communiquer avec eux!

Actuellement de nombreux problèmes sont soulevés par la situation professionnelle des ex-étudiants de langues vivantes. Ils n'ont, le plus souvent, guère envie de devenir ces enseignants à l'écart du monde économique, officiant dans un milieu clos ne communiquant qu'avec ses homologues.

Depuis un certain temps, on a dit : vous allez communiquer avec les autochtones. On a appris les langues vivantes pour voyager, pour faire du tourisme. Il est devenu aisé de faire le tour du globe sans être particulièrement aisé. Les prix des voyages baissent mais les langues n'ont pas de prix sur le marché. Après les horizons lointains, les étudiants reviennent au pays d'origine, et se retrouvent dans une situation sans horizons professionnels.

En orientant les études dans le sens des textes techniques, des traductions techniques et scientifiques, il serait possible d'apporter aux étudiants une porte de sortie vers des professions. Ceci signifie aussi qu'à côté de l'enseignement de langue proprement dit, il faudrait un enseignement de sciences reconnu dans le cursus. Quelques universités déjà suggèrent aux étudiants d'effectuer une année de médecine, de physique ou de chimie, ou toute autre spécialité scientifique qui leur permettra de se placer plus facilement sur le marché du travail.

Le besoin en traducteurs est important. Dans le domaine de la documentation technique et scientifique, des centres tels que CDST du CNRS emploient des traducteurs spécialisés ; de nombreuses firmes privées également ; aux États-Unis et au Japon les centres de documentation sont extrêmement importants ; ils vont se développer encore dans tous les pays. Il faudra de plus en plus de traducteurs pour satisfaire une demande grandissante. Actuellement, on ne sait pas toujours où les recruter. Si on prend un technicien, il ne connaît pas toujours bien les langues ; si on prend un "linguiste ", il ne connaît pas, en général, les techniques.

L'évolution de l'université devrait tenir compte de cette situation paradoxale et implanter de nouvelles filières en grand nombre. L'enseignement secondaire devrait également en tenir compte dans les programmes proposés aux élèves.

3. Si l'enseignement veut tenir compte des nouveaux besoins, il devra aussi tenir compte des nouveaux moyens.

Il peut sembler inutile de rappeler encore ici que la plupart des étudiants ne savent pas - ou n'ont pas l'habitude de taper à la machine (et les enseignants ne sont pas innocents de cette lacune : acceptent-ils toujours des devoirs tapés à la machine ?). À plus forte raison ne savent-ils pas en général se servir d'un clavier d'ordinateur. $\grave{A}$ vrai dire les choses semblent être en train de changer - avec la prolifération des jeux électroniques.

L'enseignement devrait tenir compte des instruments de traitement de texte, outils dont chacun aura fort probablement besoin dans sa vie professionnelle.

De façon plus particulière, l'enseignement de la traduction devrait actuellement tenir compte des possibilités offertes par les systèmes de traduction automatique. Les 
étudiants pourraient être formés à les connaître, à les employer, à les critiquer et à les améliorer dans les meilleurs des cas.

On se souviendra qu'avant-guerre, l'interprète de conférence n'était pas généralisé ; il ne devint présent à toutes les réunions internationales qu'après la Seconde Guerre mondiale. C'est une évolution sur laquelle personne ne songerait à revenir. De la même façon, l'ordinateur de traitement de texte, et les programmes de traduction automatique deviendront chose courante d'ici peu - et il n'y aura pas de marche arrière possible - . Ceci est dû essentiellement à l'augmentation du nombre de documents à traduire, tant dans les institutions nationales que dans les institutions internationales, documents pour la plupart techniques ou scientifiques. (La traduction littéraire, pour laquelle l'ordinateur ne sera jamais adapté, se maintenant à un niveau, c'est-à-dire n'offrant pas de débouchés suffisants à tous les étudiants de langues vivantes.)

4. Actuellement le traducteur doit produire. Il doit aussi, bien sûr, réfléchir sur ce qu'il fait. La réflexion théorique est certes très intéressante du point de vue universitaire, mais d'un point de vue commercial, elle n'est pas, en général, monnayable. Il faut produire pour vendre. Et c'est bien normal : la traduction est un produit de consommation. La diffusion grandissante des connaissances techniques et scientifiques devrait amener la traduction à devenir un produit de consommation de masse. Du point de vue du traducteur-fournisseur, il faut vendre pour vivre.

5. Concrètement, que pouvons-nous suggérer aux responsables de formation? aux enseignants de langues vivantes?

a) Tout d'abord il faudrait promouvoir une information sur ce qui se fait ailleurs dans le monde, dans les autres universités, sur ce qui se fait en matière de traduction. Par exemple à Washington, il existe un cursus de linguistique contrastive incluant la traduction automatique - en liaison avec le système SYSTRAN utilisé par la Pan American Health Organization. En France, on dissocie les institutions, on met des frontières entre enseignement et pratique, institutions universitaires et autres institutions politiques par exemple. Pourtant il existe une communauté d'intérêts entre ces entités.

b) S'informer sur les domaines les plus fructueux pour la traduction, c'est-à-dire les domaines où une documentation massive existe et où les besoins en traduction sont importants. Ceci permettrait de créer des filières spécialisées françaises, et de promouvoir un enseignement de masse pour une traduction de masse. Le recensement de ce genre de domaines est à faire. Trouvera-t-on que les plus gros fournisseurs en documentation sont les industries pharmaceutiques, les recherches en physique ou en chimie, l'énergie - et son économie -, l'environnement et ses institutions...?

c) Travailler avec des sorties-machine. Les enseignants pourraient utiliser des traductions automatiques dans leurs versions brutes pour faire travailler les étudiants : les faire réfléchir aux aspects stylistiques des textes de spécialité, et en même temps les préparer aux activités de postédition qui pourraient être l'un de leurs débouchés dans la vie professionnelle. Certains déjà, mais trop rares, le font en vue d'attirer l'attention des étudiants sur les spécificités des langages techniques et scientifiques ${ }^{2}$. On pourrait aller plus loin en pratiquant les exercices sur traduction automatique de façon systématique.

d) Ceci signifierait aussi former les étudiants (et d'abord les enseignants) à adopter une nouvelle attitude vis-à-vis des textes. Devant des textes spécialisés, il s'agit non plus de considérer l'élégance mais le contenu informationnel. Et la traduction doit rendre compte de l'information contenue dans le texte. Ni plus ni moins. Bien entendu, cette information obéit à des règles linguistiques propres à chaque langue, et on ne peut éviter le respect des règles, des normes, des usages de chaque groupe linguistique. Cependant on peut centrer son intérêt sur la transmission de l'information, et sur la fonc- 
tion du message. La fonction dicte le style, et le style n'est pas une valeur indépendante de la situation où le texte est utilisé.

En conclusion, nous dirons que la situation actuelle se présente comme une vaste course à l'adaptation. Qui s'adapte à qui ? Qui s'adapte à quoi ?

On a souvent l'impression que la technique progresse indépendamment de l'homme. Les étudiants s'adaptent (tant bien que mal) au marché professionnel. Les enseignants s'adaptent aux besoins des étudiants. Chacun s'adapte à l'envahissement de l'ordinateur. L'ordinateur peut s'adapter aux besoins des enseignants (et des étudiants) pourvu qu'on y réfléchisse et qu'au lieu de le considérer comme un monstre sacré, ou un sacré monstre, on le manipule comme n'importe lequel des ustensiles quotidiens (auxquels l'individu s'est déjà adapté, ne serait-ce que le téléphone ou l'automobile qui ont dû en faire frémir plus d'un il y a quelques décennies).

En traduction automatique, les systèmes qui sont actuellement opérationnels sont pour la plupart d'origine américaine (adaptés en Europe) ou japonais. La France va-telle s'adapter aux nouvelles techniques importées? ou bien va-t-elle créer ses propres techniques? Les choix politiques existent dans ce domaine. Mais les choix techniques ne sont pas obligatoirement les mêmes. Et l'efficacité n'est pas une notion respectant scrupuleusement les frontières.

L'adaptation réciproque de l'informatique, de l'enseignement et de la traduction débouchera sur des applications pratiques lorsqu'on aura reconnu, diffusé et intégré l'idée de leurs liens étroits.

ANNE-MARIE LAURIAN

Notes

1. Cf. AMLL, "Vulgarisation scientifique : formulation, reformulation, traduction ", dans Langue française, $n^{\circ} 64, p p .17-33$, article dans lequel il est question de cette expérience.

2. Cf. J.L. Vidalene (1984) : Traduction automatique, analyse linguistique et didactique des langues, dans Contrastes, hors série A4, janvier, pp. 121-139. 